\title{
Stability criterion for self-similar solutions with a scalar field and those with a stiff fluid in general relativity
}

\author{
Tomohiro Harada ${ }^{1 *}$ and Hideki Maeda ${ }^{2 \dagger}$ \\ ${ }^{1}$ Astronomy Unit, School of Mathematical Sciences, Queen Mary, \\ University of London, Mile End Road, London E1 4NS, UK \\ ${ }^{2}$ Advanced Research Institute for Science and Engineering, Waseda University, Tokyo 169-8555, Japan
}

(Dated: March 23, 2021)

\begin{abstract}
A stability criterion is derived in general relativity for self-similar solutions with a scalar field and those with a stiff fluid, which is a perfect fluid with the equation of state $P=\rho$. A wide class of self-similar solutions turn out to be unstable against kink mode perturbation. According to the criterion, the Evans-Coleman stiff-fluid solution is unstable and cannot be a critical solution for the spherical collapse of a stiff fluid if we allow sufficiently small discontinuity in the density gradient field in the initial data sets. The self-similar scalar-field solution, which was recently found numerically by Brady et al. (2002 Class. Quantum. Grav. 19 6359), is also unstable. Both the flat Friedmann universe with a scalar field and that with a stiff fluid suffer from kink instability at the particle horizon scale.
\end{abstract}

PACS numbers: 04.20.Dw, 04.40.-b, 04.40.Nr

\section{INTRODUCTION}

Since the Einstein equations are simultaneous nonlinear partial differential equations, it is not easy to obtain general solutions. In this context, we can assume self-similarity and consider self-similar solutions. For example, in spherical symmetry, this assumption reduces the partial differential equations to a set of ordinary differential equations [1] and even the classification of self-similar solutions with a perfect fluid has been done 2 , 3, 4] based on the theory of dynamical systems $([5,[6]$ and references therein). Thus, the assumption of self-similarity is very powerful in finding dynamical and inhomogeneous solutions. The application of self-similar solutions is very large, including cosmological perturbations, star formation, gravitational collapse, primordial black holes [7, 8, 9], cosmological voids and cosmic censorship [10, 11, 12]. See 13] for a brief review of self-similar solutions.

In recent progress in general relativity, self-similar solutions have attracted attention not only because they are easy to obtain but also because they may play important roles in cosmological situations and/or gravitational collapse. One of the important roles of self-similar solutions is to describe asymptotic behaviours of more general solutions. This feature has been found in several models such as homogeneous cosmological models [5, 6]. In a cosmological context, the suggestion that spherically symmetric fluctuations might naturally evolve from complex initial conditions to a self-similar form has been termed the similarity hypothesis [13, 14]. The hypothesis has been also suggested to hold in more general situations, including gravitational collapse. In fact, Harada and Maeda 15] numerically found that a self-similar solution acts as an attractor in the spherically symmetric collapse of a perfect fluid with the equation of state $P=k \rho(0<k<1)$ at least for $0<k \lesssim 0.03$ (see also 16$]$ ).

Spherically symmetric self-similar solutions have been also studied in the context of critical behaviour in gravitational collapse, in which a self-similar solution is not an attractor but an intermediate attractor. The critical behaviour was numerically discovered by Choptuik [17] in the spherical system of a massless scalar field. There appears a discretely self-similar solution at the threshold of black hole formation for $p=p^{*}$, where $p$ and $p^{*}$ are the parameter of initial data sets and the critical value of the parameter, respectively. For supercritical collapse, the black hole mass $M_{\mathrm{BH}}$ follows the scaling law $M_{\mathrm{BH}} \propto\left|p-p^{*}\right|^{\gamma}$, where $\gamma \approx 0.37$ is referred to as the critical exponent. Subsequently, these findings have been confirmed by several authors (e.g. 18]). Evans and Coleman 19] studied the spherical system of a radiation fluid $(P=\rho / 3)$ and found similar phenomena although the observed critical solution is a continuously selfsimilar solution. Koike, Hara and Adachi [20] showed that the critical behaviour is understood by the intermediate behaviour around a saddle equilibrium point. This approach was also successfully applied to the spherical system of a massless scalar field to understand the critical behaviour observed by Choptuik [21]. Several authors [15, 22, 23] have extended the work to perfect fluids with the equation of state $P=k \rho(0<k \leq 1)$. The limiting case $k \rightarrow 0$ turns out to be the Newtonian critical phenomena 24]. Another case $k=1$, for which the perfect fluid is called a stiff fluid, is interesting because the stiff fluid is known to be equivalent to a massless scalar field in certain circumstances [25, 26].

\footnotetext{
* Electronic address:T.Harada@qmul.ac.uk

$\dagger$ Electronic address:hideki@gravity.phys.waseda.ac.jp
} 
Neilsen and Choptuik 23 studied the stiff-fluid system and found that the critical behaviour can be regarded as a continuous limit of that for a perfect fluid with $P=k \rho(0<k<1)$ as $k \rightarrow 1$. The critical solution is continuously self-similar and the critical exponent is given by $\gamma=0.92 \pm 0.02$. Brady et al. 26] studied the mystery of why the critical behaviour for a stiff fluid is different from that for a scalar field. See 27] for a broad review of critical phenomena in gravitational collapse.

The present article concerns weak discontinuity around self-similar solutions. It has been known that the required differentiability condition for the spacetime metric tensor can be much weaker than $C^{\infty}$, for the existence and uniqueness of geodesics and/or well-defined initial value formulation of the Einstein equation [28]. Moreover, it has been well established that shock waves, which is one of the examples of strong discontinuity, play essential roles in many astrophysical situations. If we insert a perturbation with a sufficiently small weak discontinuity, it may grow too large to neglect as time proceeds. This instability has been called kink instability. The kink instability of self-similar solutions was studied by Ori and Piran 29] for the spherical system of isothermal gas in Newtonian gravity. Harada [30] (paper I) investigated the kink instability of self-similar solutions for the spherical system of a perfect fluid with the equation of state $P=k \rho(0<k<1)$ in general relativity. In this article we derive a stability criterion against the kink mode perturbation for both self-similar solutions with a scalar field and those with a stiff fluid in general relativity. Several interesting applications are discussed, one of which is related to critical behaviour for these two systems.

This article is organized as follows. In Sec. II, we derive the basic equations for the spherically symmetric Einsteinscalar-field system and those for self-similar solutions. Also in this section we introduce the kink mode for the self-similar scalar-field solutions, derive the equations for this mode and obtain the stability criterion for self-similar scalar-field solutions. In Sec. III, the analysis in paper I is extended to include the stiff-fluid system. Section IV is devoted to obtain the correspondence relation between self-similar solutions of the two systems, the scalar-field system and the stiff-fluid one. In Sec. V, we discuss the applications of the present result, in particular, to critical behaviour of gravitational collapse. In Sec. VI, we summarize the paper. We adopt units such that $G=c=1$ and the abstract index notation of [31].

\section{STABILITY CRITERION FOR SELF-SIMILAR SOLUTIONS WITH A SCALAR FIELD}

\section{A. Equations for a self-gravitating massless scalar field}

In this section, we basically follow the notation given by Brady et al. [26]. We consider a massless scalar field $\phi$ as a matter field, for which the stress-energy tensor is given by

$$
T^{a b}=\nabla^{a} \phi \nabla^{b} \phi-\frac{1}{2} g^{a b}\left(\nabla_{c} \phi \nabla^{c} \phi\right) .
$$

We adopt the Bondi coordinates for a spherically symmetric spacetime as

$$
d s^{2}=-g \bar{g} d u^{2}-2 g d u d R+R^{2}\left(d \theta^{2}+\sin ^{2} \theta d \phi^{2}\right),
$$

where $g=g(u, R)$ and $\bar{g}=\bar{g}(u, R)$. Then the Einstein equation and the equation of motion for the scalar field reduce to the following partial differential equations:

$$
\begin{aligned}
(\ln g)_{, R} & =4 \pi R \phi_{, R}^{2}, \\
R \bar{g}_{, R} & =g-\bar{g}, \\
g\left(\frac{\bar{g}}{g}\right)_{, u} & =8 \pi R\left(\phi_{, u}^{2}-\bar{g} \phi_{, u} \phi_{, R}\right), \\
\left(R^{2} \bar{g} \phi_{, R}\right)_{, R} & =2 R \phi_{, u}+2 R^{2} \phi_{, u R},
\end{aligned}
$$

where the comma denotes the partial derivative. For later convenience we define a new function $\bar{h}(u, R)$ as

$$
\phi=\bar{h}(u, R)-\kappa \ln |u|,
$$

where $\kappa$ is an arbitrary constant, and we define the following variables:

$$
x \equiv-\frac{R}{u}, \quad X \equiv \ln |x|, \quad T \equiv-\ln |u| .
$$


Then equations (2.3)-(2.6) reduce to

$$
\begin{aligned}
(\ln g)^{\prime} & =4 \pi \bar{h}^{\prime 2} \\
\bar{g}^{\prime} & =g-\bar{g} \\
g\left[\left(\frac{\bar{g}}{g}\right)+\left(\frac{\bar{g}}{g}\right)^{\prime}\right] & =8 \pi\left(\dot{\bar{h}}+\bar{h}^{\prime}+\kappa\right)\left[x\left(\dot{\bar{h}}+\bar{h}^{\prime}+\kappa\right)-\bar{g} \bar{h}^{\prime}\right], \\
\left(\bar{g} \bar{h}^{\prime}\right)^{\prime}+\bar{g} \bar{h}^{\prime} & =2 x\left[\left(\dot{\bar{h}}+\bar{h}^{\prime}\right)+\left(\dot{\bar{h}}+\bar{h}^{\prime}\right)^{\prime}+\kappa\right],
\end{aligned}
$$

where the dot and prime denote the partial derivatives with respect to $T$ and $X$, respectively. We refer to $u<0$ and $u>0$ as early times and late times, respectively. We refer to $u<0$ and $u>0$ as early times and late times, respectively. It is noted that the present definition of prime is different from that in Brady et al. [26].

\section{B. Self-similar scalar-field solutions}

For self-similar solutions, we assume that $g=g(X), \bar{g}=\bar{g}(X)$ and $\bar{h}=\bar{h}(X)$. The ordinary differential equations for $g, \bar{g}$ and $\bar{h}$ are given by

$$
\begin{aligned}
(\ln g)^{\prime} & =4 \pi j^{2}, \\
\bar{g}^{\prime} & =g-\bar{g}, \\
g-\bar{g} & =4 \pi\left[2 \kappa^{2} x-(\bar{g}-2 x)\left(j^{2}+2 \kappa j\right)\right], \\
\bar{h}^{\prime} & =j \\
(2 x-\bar{g}) j^{\prime} & =-2 \kappa x+j(g-2 x),
\end{aligned}
$$

where the prime denotes the differentiation with respect to $X$.

These equations are singular when $\bar{g}=2 x$, which is called a similarity horizon. We denote the value of $x$ at the similarity horizon as $x_{\mathrm{s}}$ and also the value of $X$ as $X_{s} \equiv \ln \left|x_{s}\right|$. This line corresponds to a radial ingoing (outgoing) null curve for early-time (late-time) solutions because

$$
\frac{d X}{d T}=1-\frac{\bar{g}}{2 x}
$$

along a radial ingoing (outgoing) null curve. We consider self-similar scalar-field solutions with finite values of functions $g, \bar{g}, \bar{h}$ and $j$ and their gradients with respect to $X$ at the similarity horizon. Then we find at $X=X_{\mathrm{s}}$

$$
g=2 x_{\mathrm{s}}\left(4 \pi \kappa^{2}+1\right), \quad \bar{g}=2 x_{\mathrm{s}}, \quad j=\frac{1}{4 \pi \kappa},
$$

for $\kappa \neq 0$ and

$$
g=\bar{g}=2 x_{\mathrm{s}}, \quad j=j_{\mathrm{s}}
$$

for $\kappa=0$. For $\kappa=0$, similarity horizons are parametrized by $j_{\mathrm{s}}$. Since we have

$$
\left(1-\frac{\bar{g}}{2 x}\right)^{\prime}=1-4 \pi \kappa^{2}
$$

at the similarity horizon, the self-similarity horizon is "transluminal", i.e. it has subluminal interior and superluminal exterior for $4 \pi \kappa^{2}<1$, while it is "anti-transluminal", i.e. it has superluminal interior and subluminal exterior for $4 \pi \kappa^{2}>1$.

We can have regular centre at $R=0$ for $u \neq 0$, which corresponds to $X=-\infty$. The regularity condition reduces to the following initial condition at $X=-\infty$.

$$
g=\bar{g}=g_{0}, \quad \bar{h}=\bar{h}_{0}, \quad j=0 .
$$

Taking the limit $X \rightarrow-\infty$ in Eq. (2.17) and using the l'Hospital's theorem, we have

$$
\lim _{X \rightarrow-\infty} \frac{j^{\prime}(X)}{x(X)}=\lim _{X \rightarrow-\infty} \frac{j(X)}{x(X)}=\frac{\kappa}{g_{0}} .
$$


The above system of Eqs. (2.13) - 2.17) was studied by Christodoulou 32], Brady 33 and Brady et al. 26]. Exact self-similar scalar-field solutions discovered by Roberts 34] correspond to general solutions for $\kappa=0$, which will be described in some detail in Appendix A Christodoulou [32] found that there is a solution with $4 \pi \kappa^{2}=1 / 3$, which has both regular centre and analytic similarity horizon and turns out to be the flat Friedmann solution. Using a two-point shooting method, Brady et al. [26] numerically found another self-similar scalar-field solution with both regular centre and analytic similarity horizon, which we will discuss in Secs. $\mathrm{VA}$ and $\mathrm{VB}$ in detail.

\section{Kink instability of self-similar solutions with a scalar field}

We consider perturbations which satisfy the following conditions in the background of a self-similar solution: (1) The initial perturbations vanish inside the similarity horizon for early-time solutions $(u<0)$. (For late-time solutions $(u>0)$, the initial perturbations vanish outside the similarity horizon.) (2) $g, \bar{g}, \bar{h}$ and $\bar{h}^{\prime}$ are continuous everywhere, in particular at the similarity horizon. (3) $\bar{h}^{\prime \prime}$ and $\dot{\bar{h}}^{\prime \prime}$ are discontinuous at the similarity horizon, although they have finite one-sided limit values as $X \rightarrow X_{\mathrm{s}}-0$ and $X \rightarrow X_{\mathrm{s}}+0$.

We denote the full-order perturbations as

$$
\delta g(T, X) \equiv g(T, X)-g_{\mathrm{b}}(X), \quad \delta \bar{g}(T, X) \equiv \bar{g}(T, X)-\bar{g}_{\mathrm{b}}(X), \quad \delta \bar{h}(T, X) \equiv \bar{h}(T, X)-\bar{h}_{\mathrm{b}}(X),
$$

where $g_{\mathrm{b}}, \bar{g}_{\mathrm{b}}$ and $\bar{h}_{\mathrm{b}}$ denote the background self-similar solution.

By conditions (2), the perturbations satisfy $\delta \bar{h}=\delta \bar{h}^{\prime}=0$ and $\delta \bar{h}^{\prime \prime} \neq 0$ at the similarity horizon at initial moment $T=T_{0}$. The evolution of the initially unperturbed region is completely described by the background self-similar solution because no information from the perturbed side can penetrate the unperturbed side by condition (1). Then, by conditions (2) and (3), we find $\delta \bar{h}=\delta \bar{h}^{\prime}=0$ and $\delta \bar{h}^{\prime \prime} \neq 0$ at the similarity horizon for $T \geq T_{0}$ for early-time solutions (for $T \leq T_{0}$ for late-time solutions). We find $\delta g=\delta \bar{g}=\delta g^{\prime}=\delta \bar{g}^{\prime}=\delta \bar{g}^{\prime \prime}=0$ but $\delta g^{\prime \prime} \neq 0$ and $\delta \bar{g}^{\prime \prime \prime} \neq 0$ from Eqs. (2.9), (2.10) and (2.11) for the full-order perturbations at the point of discontinuity.

Differentiating Eq. 2.9 with respect to $X$ and estimating both sides at the point of discontinuity, we obtain

$$
\delta g^{\prime \prime}=8 \pi g_{\mathrm{b}} j_{\mathrm{b}} \delta j^{\prime}
$$

Using Eqs. (2.9) and (2.10), differentiating Eq. (2.11) with respect to $X$, and estimating both sides at the point of discontinuity, we obtain

$$
\left(j_{\mathrm{b}}+\kappa\right)\left(\bar{g}_{\mathrm{b}}-2 x\right) \delta j^{\prime}=0 .
$$

Therefore, $\delta j^{\prime} \neq 0$ is possible at the similarity horizon $X=X_{\mathrm{s}}$ where $\bar{g}_{\mathrm{b}}=2 x_{\mathrm{s}}$.

Using Eqs. (2.9), (2.10) and (2.26), differentiating Eq. (2.11) twice with respect to $X$, and estimating both sides at the point of discontinuity, we obtain

$$
2 x\left(j_{\mathrm{b}}+\kappa\right) \delta \dot{j}^{\prime}-\left[g_{\mathrm{b}} j_{\mathrm{b}}+2\left(g_{\mathrm{b}}-4 x\right)\left(j_{\mathrm{b}}+\kappa\right)\right] \delta j^{\prime}=\left(j_{\mathrm{b}}+\kappa\right)\left(\bar{g}_{\mathrm{b}}-2 x\right) \delta j^{\prime \prime} .
$$

We can have another equation by using Eq. (2.10), differentiating Eq. (2.12) with respect to $X$, and estimating both sides at the point of discontinuity as

$$
2 x \delta \dot{j}^{\prime}-\left(2 g_{\mathrm{b}}-\bar{g}_{\mathrm{b}}-4 x\right) \delta j^{\prime}=\left(\bar{g}_{\mathrm{b}}-2 x\right) \delta j^{\prime \prime} .
$$

The two equations obtained above are independent in general but degenerate at the similarity horizon. At the similarity horizon, we find that these two equations reduce to

$$
\delta \dot{j}^{\prime}-\left(8 \pi \kappa^{2}-1\right) \delta j^{\prime}=\lim _{X \rightarrow X_{\mathrm{s}}}\left[\left(\frac{\bar{g}_{\mathrm{b}}}{2 x}-1\right) \delta j^{\prime \prime}\right]
$$

where Eq. (2.19) or Eq. (2.20) is used. Since the left-hand side has a finite limit at the similarity horizon, the righthand side also has a finite limit. Since $\delta j^{\prime \prime}$ may not have a finite limit at the similarity horizon, the limit value on the right-hand side is not trivial. However, we can show that the right-hand side vanishes employing the proof in Appendix [B] Therefore, we obtain the full-order perturbation equation for $\delta j^{\prime}$ at the similarity horizon:

$$
\delta \dot{j}^{\prime}-\left(8 \pi \kappa^{2}-1\right) \delta j^{\prime}=0
$$

It should be noticed that the full-order analysis results in a linear-order equation. This equation can be integrated as

$$
\delta j^{\prime}=\text { const } \cdot e^{\alpha T}
$$


where

$$
\alpha=-\left(1-8 \pi \kappa^{2}\right)
$$

Therefore, for early-time solutions, it is found that the perturbation decays exponentially for $4 \pi \kappa^{2}<1 / 2$, it is constant for $4 \pi \kappa^{2}=1 / 2$ and it grows exponentially for $4 \pi \kappa^{2}>1 / 2$. The situation is reversed for late-time solutions.

Here we define instability by the exponential growth of discontinuity. Then we find the following criterion: for early-time solutions, solutions with a regular similarity horizon and $4 \pi \kappa^{2}<1 / 2$ are stable against the kink mode, while those with $4 \pi \kappa^{2}>1 / 2$ are unstable. Solutions with $4 \pi \kappa^{2}=1 / 2$ are marginally stable against this mode. The situation is reversed for late-time solutions.

\section{STABILITY CRITERION FOR SELF-SIMILAR SOLUTIONS WITH A STIFF FLUID}

\section{A. Equations for a self-gravitating stiff fluid}

In Sec. IC we have seen that the kink instability can occur in self-similar scalar-field solutions. Since a scalar field is equivalent to a stiff fluid, it is expected that self-similar stiff-fluid solutions can also have the kink instability. However, the equivalence turns out to be incomplete as we will see in Sec. IV Hence it will be interesting to see when and how the kink instability occurs in stiff-fluid self-similar solutions. In paper I, the kink instability is studied in perfect-fluid systems with the equation of state $P=k \rho(0<k<1)$. Almost all equations in paper I are available also for a stiff fluid with $k=1$. Here we avoid repetition of the derivation and only quote the equations which are necessary for the subsequent analysis. We basically follow the formulation given by [9].

The stress-energy tensor for a stiff fluid is given by

$$
T^{a b}=\rho\left(2 u^{a} u^{b}+g^{a b}\right),
$$

where $\rho$ is the energy density and $u^{a}$ is the four velocity of the fluid element. In a spherically symmetric spacetime, there is a natural quasilocal mass called the Misner-Sharp mass, defined by [35]

$$
m \equiv \frac{R}{2}\left(1-\nabla^{a} R \nabla_{a} R\right),
$$

where $R$ is the circumferential radius. The line element in a spherically symmetric spacetime is given by

$$
d s^{2}=-e^{\sigma(t, r)} d t^{2}+e^{\omega(t, r)} d r^{2}+R^{2}(t, r)\left(d \theta^{2}+\sin ^{2} \theta d \phi^{2}\right),
$$

where we choose the radial coordinate $r$ to be comoving with fluid elements. We define dimensionless functions such as

$$
\eta \equiv 8 \pi r^{2} \rho, \quad S \equiv \frac{R}{r}, \quad M \equiv \frac{2 m}{r}, \quad y \equiv \frac{M}{\eta S^{3}},
$$

where we have also defined an auxiliary function $y$ for later convenience. We introduce the following variables:

$$
\xi \equiv \frac{t}{r}, \quad \tau \equiv-\ln |t|, \quad z \equiv-\ln |\xi| .
$$

The velocity function $V$ is defined by the velocity of the $\xi=$ const curve relative to the fluid element, which is written as

$$
V=\operatorname{sign}(\xi) e^{z+\frac{\omega-\sigma}{2}} .
$$

We can find that the equations of motion for the fluid are integrated as

$$
e^{\sigma}=a_{\sigma} \eta^{-1} e^{2 z}, \quad e^{\omega}=a_{\omega} \eta^{-1} S^{-4},
$$

where we make $a_{\sigma}$ and $a_{\omega}$ constant using coordinate transformation. Using Eqs. (3.7), $V^{2}$ is rewritten as

$$
V^{2}=\frac{a_{\omega}}{a_{\sigma}} S^{-4} .
$$


Using the dimensionless variables, we can derive the following equations from the Einstein equations:

$$
\begin{aligned}
& M+M^{\prime}=\eta S^{2}\left(S+S^{\prime}\right), \\
& \dot{M}+M^{\prime}=-\eta S^{2}\left(\dot{S}+S^{\prime}\right), \\
& \ddot{S}+2 \dot{S}^{\prime}+S^{\prime \prime}+\left[\frac{1}{2}\left(\frac{\dot{\eta}}{\eta}+\frac{\eta^{\prime}}{\eta}-2\right)+1\right]\left(\dot{S}+S^{\prime}\right) \\
= & -\frac{1}{2} e^{\sigma-\omega-2 z}\left(\frac{\eta^{\prime}}{\eta}-2\right)\left(S+S^{\prime}\right)-\frac{1}{2} e^{\sigma-2 z} \frac{M+\eta S^{3}}{S^{2}},
\end{aligned}
$$

and

$$
\frac{M}{S}=1+e^{-\sigma+2 z}\left(\dot{S}+S^{\prime}\right)^{2}-e^{-\omega}\left(S+S^{\prime}\right)^{2},
$$

where $e^{\sigma}$ and $e^{\omega}$ are given by Eq. (3.7), the dot and prime denote the partial derivatives with respect to $\tau$ and $z$, respectively, and three of the above four equations are independent.

\section{B. Self-similar stiff-fluid solutions}

For self-similar solutions, we assume that all dimensionless quantities depend only on $z$, i.e., $M=M(z), S=S(z)$, $\eta=\eta(z), \sigma=\sigma(z)$, and $\omega=\omega(z)$. Then, from equations (3.9)-3.12), we obtain the following ordinary differential equations:

$$
\begin{aligned}
M^{\prime} & =\frac{1}{2} \frac{1-y}{y} M \\
S^{\prime} & =-\frac{1-y}{2} S \\
\eta^{\prime} & =2\left[(1-y)+\frac{y-a_{\sigma} V^{2}}{1-V^{2}}\right] \eta,
\end{aligned}
$$

and constraint equation

$$
V^{2}(1-y)^{2}-(1+y)^{2}+4 a_{\sigma} V^{2} y\left(\frac{S}{M}-1\right)=0,
$$

where $V^{2}$ is given by Eq. (3.8). It should be noted that $a_{\sigma}$ does not change under the scale transformation and therefore its value has a physical meaning. $a_{\sigma}$ is physically characterized by the following relation:

$$
a_{\sigma}=e^{\sigma} \eta e^{-2 z}=8 \pi \rho e^{\sigma} t^{2} .
$$

A sonic point $z=z_{\mathrm{s}}$ is defined by

$$
V^{2}=1
$$

Since the sound speed is equal to the light speed, the sonic point may correspond to the Cauchy horizon, event horizon or particle horizon, simultaneously. From Eqs. (3.8), (3.16) and (3.18), we find $M=M_{\mathrm{s}}$ and $S=S_{\mathrm{s}}$ at the sonic point, where

$$
M_{\mathrm{s}}=\frac{a_{\sigma}}{1+a_{\sigma}}\left(\frac{a_{\omega}}{a_{\sigma}}\right)^{1 / 4}, \quad S_{\mathrm{s}}=\left(\frac{a_{\omega}}{a_{\sigma}}\right)^{1 / 4} .
$$

Here we adopt the finiteness of the density gradient as a regularity condition. Then, from Eq. (3.15), we obtain $y=y_{\mathrm{s}}$ at the sonic point, where

$$
y_{\mathrm{s}}=a_{\sigma} .
$$

We introduce a new independent variable $u$, which is defined as

$$
\frac{d z}{d u}=1-V^{2}
$$


Using $u$ in place of $z$, we have the following system of the ordinary differential equations:

$$
\begin{aligned}
\frac{d M}{d u} & =\frac{1}{2} \frac{1-y}{y}\left(1-V^{2}\right) M, \\
\frac{d S}{d u} & =-\frac{1-y}{2}\left(1-V^{2}\right) S, \\
\frac{d \eta}{d u} & =2\left[(1-y)\left(1-V^{2}\right)+\left(y-a_{\sigma} V^{2}\right)\right] \eta .
\end{aligned}
$$

In the above, the sonic point is found to be an equilibrium point of the dynamical system with four dimensional state space $\{(M, S, \eta, z)\}$. More precisely, the whole of these sonic points composes the two dimensional surface of equilibrium points determined by Eqs. (3.18) and (3.20), which is called a sonic surface. The qualitative properties of solutions are analyzed by the linearisation of the ordinary differential equations at the equilibrium point. We put

$$
M=\eta_{\mathrm{s}} S_{\mathrm{s}}^{3}\left(y_{\mathrm{s}}+x_{1}\right), \quad S=S_{\mathrm{s}}\left(1+x_{2}\right), \quad \eta=\eta_{\mathrm{s}}\left(1+x_{3}\right), \quad \xi=\xi_{\mathrm{s}}\left(1+x_{4}\right) \quad\left(\text { or } \quad z=z_{s}-x_{4}\right),
$$

where $x_{1}$ to $x_{4}$ are regarded as components of the vector $\mathbf{x}$. Then, we find the following linearised ordinary differential equations in the matrix form:

$$
\frac{d}{d u} \mathbf{x}=\mathbf{A x}
$$

where the matrix $\mathbf{A}$ is given by

$$
\mathbf{A}=2\left(\begin{array}{cccc}
0 & 1-y_{\mathrm{s}} & 0 & 0 \\
0 & -\left(1-y_{\mathrm{s}}\right) & 0 & 0 \\
1 & \left(4-3 y_{\mathrm{s}}\right) & -y_{\mathrm{s}} & 0 \\
0 & -2 & 0 & 0
\end{array}\right)
$$

This matrix has two zero eigenvalues and two generically nonzero eigenvalues. The former two zero eigenvalues are due to the two dimensional surface of equilibrium points in four dimensional state space. The latter two eigenvalues are:

$$
\lambda_{1}=-2 y_{\mathrm{s}}, \quad \text { and } \quad \lambda_{2}=-2\left(1-y_{\mathrm{s}}\right),
$$

associated with the following eigenvectors:

$$
\mathbf{e}_{\mathbf{1}} \equiv\left(\begin{array}{l}
0 \\
0 \\
1 \\
0
\end{array}\right), \quad \text { and } \quad \mathbf{e}_{\mathbf{2}} \equiv\left(\begin{array}{c}
\left(1-2 y_{\mathrm{s}}\right)\left(1-y_{\mathrm{s}}\right) \\
-\left(1-2 y_{\mathrm{s}}\right)\left(1-y_{\mathrm{s}}\right) \\
3\left(1-y_{\mathrm{s}}\right)^{2} \\
-2\left(1-2 y_{\mathrm{s}}\right)
\end{array}\right)
$$

respectively. Therefore, as we restrict our attention to the plane spanned by $\mathbf{e}_{1}$ and $\mathbf{e}_{2}$, the sonic point is a node with primary direction $\mathbf{e}_{1}$ and secondary direction $\mathbf{e}_{2}$ for $0<y_{\mathrm{s}}<1 / 2$, a degenerate node for $y_{\mathrm{s}}=1 / 2$, a node with primary direction $\mathbf{e}_{2}$ and secondary direction $\mathbf{e}_{1}$ for $1 / 2<y_{\mathrm{s}}<1$, and a saddle with repulsive-eigenvalue direction $\mathbf{e}_{2}$ and attractive-eigenvalue direction $\mathbf{e}_{1}$ for $1<y_{\mathrm{s}}$.

Both eigenvectors are regular in the whole four dimensional state space $\{(M, S, \eta, z)\}$ but not in the physical space. Actually, along eigenvector $\mathbf{e}_{\mathbf{1}}$, the density gradient takes the following limit:

$$
\lim _{z \rightarrow z_{\mathrm{s}}} \frac{\eta^{\prime}}{\eta}= \pm \infty
$$

The above divergence directly implies the divergence of the density gradient with respect to the physical length $l$ on the $t=$ const hypersurface as seen in the following equation:

$$
\left(\frac{\partial \rho}{\partial l}\right)_{t}=e^{-\omega / 2}\left(\frac{\partial}{\partial r} \frac{\eta}{8 \pi r^{2}}\right)_{t}=e^{-\omega / 2} \frac{\eta}{8 \pi r^{3}}\left(\frac{\eta^{\prime}}{\eta}-2\right) .
$$

Hereafter, we restrict our attention to the self-similar solutions with finite density gradients at the sonic point. Under this restriction it is found that only the solutions along $\mathbf{e}_{2}$ are allowed and that there is no acceptable solution with 
$y_{\mathrm{s}}=1 / 2$. This is quite different from the case for $0<k<1$, in which both directions associated with the two eigenvalues are physically acceptable. Along direction $\mathbf{e}_{\mathbf{2}}$, we have

$$
\frac{S^{\prime}}{S}=-\frac{1}{2}\left(1-y_{\mathrm{s}}\right)
$$

at the sonic point for $y_{\mathrm{s}} \neq 1 / 2$. Then, from Eq. (3.8), we have

$$
\left(V^{2}\right)^{\prime}=2\left(1-y_{\mathrm{s}}\right)
$$

at the sonic point. Therefore, the sonic point is "transsonic" for $0<y_{\mathrm{s}}<1 / 2$ or $1 / 2<y_{\mathrm{s}}<1$, while the sonic point is "anti-transsonic" for $1<y_{\mathrm{s}}$.

The classification is as follows. For $0<y_{\mathrm{s}}<1 / 2$, the solution crosses a nodal sonic point along a secondary direction. For $y_{\mathrm{s}}=1 / 2$, the solution crosses a degenerate-nodal sonic point. For $1 / 2<y_{\mathrm{s}}<1$, the solution crosses a nodal sonic point along a primary direction. For $1<y_{\mathrm{s}}$, the solution crosses a saddle sonic point along a repulsive-eigenvalue direction. As for the family of acceptable solutions, for $0<y_{\mathrm{s}}<1 / 2$ or $1<y_{\mathrm{s}}$ there is only one acceptable solution which crosses the sonic point and it has analyticity there (see paper I for the analyticity of self-similar solutions at the sonic point. 36]) For $1 / 2<y_{\mathrm{s}} \leq 1$, there is a one-parameter family of acceptable solutions which crosses the sonic point and only one of them has analyticity there. For $y_{\mathrm{s}}=1 / 2$, there is no acceptable solution. It is clear that the case for $y_{\mathrm{s}}=1$ needs more careful treatment because the equilibrium point is nonhyperbolic (c.f. the Hartman-Grobman theorem). Bicknell and Henriksen [9] found that there is a one-parameter family of regular solutions in this case by a direct integration around the sonic point.

\section{Kink instability of self-similar solutions with a stiff fluid}

We consider perturbations which satisfy the following conditions in the background of a self-similar solution: (1) The initial perturbations vanish inside the sonic point for early-time solutions $(t<0)$. (The initial perturbations vanish outside the sonic point for late-time solutions $(t>0)$.) (2) $M, S$ and $\eta$ are continuous everywhere, in particular at the sonic point. (3) $\eta^{\prime}$ and $\dot{\eta}^{\prime}$ are discontinuous at the sonic point, although they have finite one-sided limit values as $z \rightarrow z_{\mathrm{s}}-0$ and $z \rightarrow z_{\mathrm{s}}+0$ [37].

We denote the full-order perturbations as

$$
\delta S(\tau, z) \equiv S(\tau, z)-S_{\mathrm{b}}(z), \quad \delta M(\tau, z) \equiv M(\tau, z)-M_{\mathrm{b}}(z), \quad \delta \eta(\tau, z) \equiv \eta(\tau, z)-\eta_{\mathrm{b}}(z),
$$

where $M_{\mathrm{b}}, S_{\mathrm{b}}$ and $\eta_{\mathrm{b}}$ denote the background self-similar solution.

For the perfect fluid case for the equation of state $P=k \rho$, the evolution equation for the discontinuity at the sonic point becomes (paper I but [38])

$$
\frac{\delta \eta^{\prime}}{\eta_{\mathrm{b}}}+\left(-2 \frac{1-k}{1+k} \frac{\eta_{\mathrm{b}}^{\prime}}{\eta_{\mathrm{b}}}+\frac{5-3 k-4 y_{\mathrm{s}}}{1+k}\right) \frac{\delta \eta^{\prime}}{\eta_{\mathrm{b}}}-\frac{1-k}{1+k}\left(\frac{\delta \eta^{\prime}}{\eta_{\mathrm{b}}}\right)^{2}=\lim _{z \rightarrow z_{s}}\left[\frac{1}{2}\left(\frac{k}{V^{2}}-1\right) \frac{\delta \eta^{\prime \prime}}{\eta_{\mathrm{b}}}\right]
$$

where the finite limit value on the right-hand side exists because the left-hand side has a finite limit value. Employing the proof given in Appendix $[\mathrm{B}$, we can show that the limit value on the right-hand side is zero. Then, we have (Eq.(4.15) of paper I)

$$
\frac{\delta \dot{\eta}^{\prime}}{\eta_{\mathrm{b}}}+\left(-2 \frac{1-k}{1+k} \frac{\eta_{\mathrm{b}}^{\prime}}{\eta_{\mathrm{b}}}+\frac{5-3 k-4 y_{\mathrm{s}}}{1+k}\right) \frac{\delta \eta^{\prime}}{\eta_{\mathrm{b}}}-\frac{1-k}{1+k}\left(\frac{\delta \eta^{\prime}}{\eta_{\mathrm{b}}}\right)^{2}=0 .
$$

With $k=1$, we find that the equation for full-order perturbation at the sonic point is given by

$$
\frac{\delta \dot{\eta^{\prime}}}{\eta_{\mathrm{b}}}+\left(1-2 y_{\mathrm{s}}\right) \frac{\delta \eta^{\prime}}{\eta_{\mathrm{b}}}=0
$$

It should be noticed that the full-order analysis results in a linear-order equation. This equation is integrated as

$$
\frac{\delta \eta^{\prime}}{\eta_{\mathrm{b}}}=\mathrm{const} \cdot e^{\alpha \tau}
$$

where

$$
\alpha \equiv-\left(1-2 y_{\mathrm{s}}\right)
$$


Therefore, it is found that as $\tau$ increases, the discontinuity in $\eta^{\prime}$ decays exponentially for $0<y_{\mathrm{s}}<1 / 2$ but grows exponentially for $1 / 2<y_{\mathrm{s}}$. We should note that the parameter $y_{\mathrm{s}}$ can be identified with $a_{\sigma}$ by the discussion given in Sec. IIIB

This result for the stiff-fluid case is quite different from that for $0<k<1$ in the following respect. For the latter case, the instability results in the divergence of $\delta \eta^{\prime}$ for a finite value of $\tau$ because of its nonlinear growth. However, for the former stiff-fluid case, the instability is much weaker and only exponential with respect to $\tau$. This weakness of kink instability for the stiff-fluid case may make the physical interpretation of instability rather subtle as is mentioned in paper I. However, here we define instability by the exponential growth of discontinuity.

Then we find the following criterion: for early-time solutions, solutions with a regular sonic point and $0<y_{\mathrm{s}}<1 / 2$ are stable against the kink mode, while those with a regular sonic point and $1 / 2<y_{\mathrm{s}}$ are unstable against the kink mode. The situation is reversed for late-time solutions. If the criterion for kink instability should be related to the classification of sonic points, we can give the following criterion: as for early-time solutions, nodal-point secondary-direction solutions are stable, while nodal-point primary-direction solutions and saddle-point, repulsiveeigenvalue-direction solutions are unstable. The situation is reversed for late-time solutions. The above indicates that the stability criterion in terms of the properties of sonic points, which is obtained in paper I for $0<k<1$, also applies to the stiff-fluid case $k=1$.

\section{CORRESPONDENCE BETWEEN SCALAR-FIELD AND STIFF-FLUID SOLUTIONS}

\section{A. Correspondence between a scalar field and a stiff fluid}

We briefly review the correspondence between a scalar field and a stiff fluid. The stress-energy tensor for the stiff fluid is written by Eq. (3.1). We assume $\rho>0$. Introducing a scalar field $\phi$ with a future-pointing timelike gradient such as

$$
\nabla_{a} \phi=\sqrt{2 \rho} u_{a}
$$

which is possible only for an irrotational velocity field, we have

$$
\begin{aligned}
\rho & =-\frac{1}{2} \nabla_{c} \phi \nabla^{c} \phi, \\
u_{a} & =\frac{\nabla_{a} \phi}{\sqrt{-\nabla_{c} \phi \nabla^{c} \phi}},
\end{aligned}
$$

and it turns out that the stress-energy tensor is written by Eq. (2.1), i.e. that of a massless scalar field. Therefore, the irrotational stiff fluid with positive energy density has an equivalent scalar field. Actually, the correspondence is not one to one but one stiff-fluid solution to two scalar-field solutions because $\tilde{\phi}=-\phi$ is also an equivalent solution changing the relation (4.1) as

$$
-\nabla_{a} \tilde{\phi}=\sqrt{2 \rho} u_{a} .
$$

Inversely, the scalar-field solution has an equivalent physical stiff fluid only if it has a timelike gradient.

\section{B. Correspondence between self-similar scalar-field solutions and self-similar stiff-fluid solutions}

Here we obtain the correspondence between the self-similar scalar-field solutions studied in Sec. [IB in the Bondi coordinates and the self-similar stiff-fluid solutions studied in Sec IIIB in the comoving coordinates.

In the Bondi coordinates (2.2), Eq. (4.2) yields

$$
\rho=\frac{1}{2 g} \phi_{, R}\left(2 \phi_{, u}-\bar{g} \phi_{, R}\right)=\frac{1}{2 R^{2}} \frac{j}{g}[2 x(j+\kappa)-\bar{g} j] .
$$

The relation between the time coordinates $t$ and $u$ is given by the two equivalent expressions for the proper time element at the regular centre as

$$
e^{\sigma / 2}(-\infty) d t=\sqrt{g \bar{g}}(-\infty) d u
$$


where the arguments of the left-hand and right-hand sides are $z$ and $X$, respectively. Integrating the above equation, we have

$$
e^{\sigma / 2}(-\infty) t=\sqrt{g \bar{g}}(-\infty) u .
$$

Then we can write the parameter $a_{\sigma}$ of stiff-fluid solutions in terms of the quantities of scalar-field solutions as

$$
\begin{aligned}
a_{\sigma} & =\lim _{z \rightarrow-\infty} \eta e^{\sigma} e^{-2 z}, \\
& =\lim _{r \rightarrow 0} 8 \pi \rho(t, r) r^{2} e^{\sigma}\left(\frac{-t}{r}\right)^{2}, \\
& =\lim _{R \rightarrow 0} 8 \pi \rho(u, R) R^{2} g \bar{g}\left(\frac{-u}{R}\right)^{2}, \\
& =\lim _{X \rightarrow-\infty} 4 \pi x^{-2} \bar{g} j[2 x(j+\kappa)-\bar{g} j] .
\end{aligned}
$$

Therefore, the relation between the self-similar stiff-fluid solution and scalar-field solution is given by

$$
a_{\sigma}=4 \pi \kappa^{2},
$$

where Eqs. (2.22) and (2.23) are used.

Moreover, we can explicitly show that the stiff-fluid solution which is equivalent to the scalar-field solution with regular centre and regular similarity horizon satisfies the regularity condition at the sonic point. The definition of the Misner-Sharp mass yields

$$
m=\frac{R}{2}\left(1-\frac{\bar{g}}{g}\right)
$$

Then the function $y$ is written in terms of the quantities associated with the scalar-field solution as

$$
y=\frac{m}{4 \pi \rho R^{3}}=\frac{g-\bar{g}}{4 \pi j[2 x(j+\kappa)-\bar{g} j]} .
$$

Then we obtain

$$
y_{\mathrm{s}}=4 \pi \kappa^{2},
$$

at the sonic point, where Eqs. (2.19) or (2.20) are used. Therefore, the regularity condition (3.20) at the sonic point for stiff-fluid solutions is satisfied.

Equation (4.11) is a crucial correspondence relation between a self-similar scalar-field solution and self-similar stiff-fluid solution. As expected, when there is the equivalence between a self-similar scalar-field solution and a selfsimilar stiff-fluid solution, the stability against the kink mode coincides for both self-similar solutions, as is seen from Eqs. (2.31), (2.32), (3.38) and (3.39). The growth rates of the kink mode are also the same.

\section{APPLiCATIONS}

\section{A. Evans-Coleman stiff-fluid solution and critical behaviour}

There are two important sequences of numerical self-similar solutions with analyticity for a perfect fluid with $P=k \rho$, the one is the Larson-Penston solution and the other is the Evans-Coleman solution. The Larson-Penston solution in general relativity was first discovered and discussed in the context of cosmic censorship by Ori and Piran [10], and further analysis was done [11, 12, 30]. The solution crosses a node along a secondary direction for $0<k \lesssim 0.036$, a degenerate node for $k \approx 0.036$, and a node along a primary direction for $0.036 \lesssim k<1 / 3$. However, the existence of the sequence of Larson-Penston solutions for a stiff fluid has not been demonstrated yet. Actually, in our preliminary numerical survey, the existence of the Larson-Penston solution was confirmed only for $0<k<1 / 3$.

The Evans-Coleman solution was first discovered by Ori and Piran [1], identified with a critical solution by Evans and Coleman for $k=1 / 3$, and shown to exist and identified with a critical solution for $0<k \leq 1$ by several authors [22, 23, 26]. Carr et al. [4] indicated that the character of the sonic point for the Evans-Coleman solution changes as follows: the solution crosses a saddle along an attractive-eigenvalue direction for $0<k \lesssim 0.41$, a node 
along a secondary direction for $0.41 \lesssim k \lesssim 0.89$, a degenerate node for $k \approx 0.89$, and a node along a primary direction for $0.89 \lesssim k<1$.

For the scalar-field system, Brady et al. 26] numerically solved the set of ordinary differential equations (2.13)(2.17) and searched the value of $\kappa$ for which the solution has regular centre and analytic similarity horizon. They found that there is such a solution with $4 \pi \kappa^{2} \approx 0.577$. We refer to this self-similar solution as the Brady-ChoptuikGundlach-Neilsen (BCGN) solution. They found that this solution has a single unstable mode which is analytic at the similarity horizon, and that no physical stiff fluid can reproduce this solution because the BCGN solution has a spacelike hypersurface $X=X_{1}\left(>X_{\mathrm{s}}\right)$, on which the gradient of the scalar field is null and beyond which it is spacelike. They mentioned that it is possible to consider another continuation beyond $X=X_{1}$ and then the solution can be regarded as a physical stiff-fluid solution. Although this solution has some discontinuity at $X=X_{1}$, it can be considered as a continuous limit of the Evans-Coleman perfect-fluid solution with $P=k \rho$ for $0.89 \lesssim k<1$ (see Fig. 5 of [26]). Hence we refer to this solution as the Evans-Coleman stiff-fluid solution. Neilsen and Choptuik 23]'s numerical simulation suggests that this solution would be a critical solution of stiff-fluid gravitational collapse at the threshold of black hole formation (see Fig. 2 of [26]).

First we consider the stability of the Evans-Coleman stiff-fluid solution. The parameter for this solution is given by $a_{\sigma}=y_{\mathrm{s}} \approx 0.577$ using the correspondence relations (4.8) and (4.11). Then, based on the analysis in Sec. IIIB the sonic point of the Evans-Coleman stiff-fluid solution is a node and the solution crosses along a primary direction. This is quite reasonable for a $k=1$ Evans-Coleman solution since the Evans-Coleman solution crosses a node along a primary direction for $k \rightarrow 1$. From the stability criterion obtained in Sec. IIIC the Evans-Coleman stiff-fluid solution is unstable against the kink mode because $y_{\mathrm{s}} \simeq 0.577>1 / 2$. Actually, the stability against the kink mode does not change whatever continuation one chooses beyond $X=X_{1}>X_{\mathrm{s}}$. The Evans-Coleman stiff-fluid solution has two unstable modes, one is analytic and the other is kink. This implies that this solution cannot be a critical solution of stiff-fluid gravitational collapse if we allow initial data with sufficiently small discontinuity in the density gradient field. It is discussed in paper I why the kink instability did not affect Neilsen and Choptuik [23]'s fluid simulation.

\section{B. BCGN scalar-field solution and critical behaviour}

Based on the analysis in Sec. IIC the BCGN scalar-field solution is unstable against the kink mode because $4 \pi \kappa^{2} \simeq 0.577>1 / 2$. The BCGN scalar-field solution has two unstable modes, one is analytic and the other is kink. This implies that this solution cannot be a critical solution of scalar-field gravitational collapse if we allow initial data with sufficiently small discontinuity in the second-order derivative of the scalar field.

Since this solution was found to have a single analytic unstable mode, this solution is expected to act as an intermediate attractor at least locally for some critical behaviour related to the asymptotic behaviour of gravitational collapse from the point of view proposed by [20] if we restrict our attention to evolution with $C^{\infty}$ functions for instance. However, no numerical simulation has been reported so far, that suggests the existence of critical behaviour associated with this solution. Brady et al. [26] stated that the BCGN scalar-field solution is disqualified as a critical solution at the threshold of black hole formation because it has an apparent horizon beyond the similarity horizon. Since Choptuik [17]'s numerical simulation breaks down at the formation of an apparent horizon because of the choice of coordinate system, it would be reasonable that the critical behaviour associated with the BCGN scalar-field solution was not observed in his calculation. However, Hamadé and Stewart 18] did not mention the observation of critical behaviour associated with a continuously self-similar solution like the BCGN scalar-field solution, although their numerical simulation is based on the double-null formulation, which can treat an apparent horizon without any coordinate singularity.

Here we would like to speculate about this puzzle, discrete self-similarity or continuous self-similarity. Of course, we should note the possibility that the numerical survey may not have been sufficiently complete yet. If this possibility is true, further numerical investigation based on the double-null formulation would reveal a hidden critical behaviour associated with the continuously self-similar solution, i.e. the BCGN scalar-field solution, for which the critical exponent should be given by $\gamma=0.92 \pm 0.02$. This critical behaviour associated with continuous self-similarity would be seen not at the black hole threshold but inside the apparent horizon. In other words, it would be hidden by an apparent horizon and therefore by an event horizon and could not be observed by a distant observer. We would have two distinct critical behaviours for the same system, one is associated with discrete self-similarity and the other is associated with continuous self-similarity. However, we can suggest another possibility that the kink instability, which we have analysed here, may prevent the BCGN solution from involving critical behaviour. The possibility depends on which class of initial data sets we regard as physically realistic. Mathematically one can avoid the kink instability by restricting oneself to solutions in $C^{\infty}$ class. In numerical simulations, however, the differentiability condition may cause some subtle problems. The above speculations can be proved or disproved by further numerical investigations. In each case, it is inferred that the qualitative properties of dynamical solutions in the spherical system of a massless 
scalar field would be much more complicated than usually supposed in the context of critical behavior.

\section{Exact self-similar solutions}

For the flat Friedmann solution, we find $4 \pi \kappa^{2}=1 / 3$ as a scalar-field solution and $a_{\sigma}=y=1 / 3=$ const as a stiff-fluid solution. It is found that this solution is stable for early times (collapsing phase) but unstable for late times (expanding phase). This implies that there exists kink instability at the particle horizon scale for the flat Friedmann scalar-field universe and also for the flat Friedmann stiff-fluid universe.

The Roberts solutions are exact and general self-similar solutions for the scalar-field system with $\kappa=0$, which will be described in Appendix A According to the stability criterion obtained here, these solutions do not suffer from kink instability for early times while they do for late times. Since this solution has a null gradient of the scalar field at the similarity horizon, the stability criterion for self-similar stiff-fluid solutions is not applicable to this solution.

We can find the static self-similar solution with a scalar field for $4 \pi \kappa^{2}=1$, which is given by

$$
\bar{g}=2 x, \quad g=4 x, \quad \bar{h}= \pm(4 \pi)^{-1 / 2} \ln |x|+\text { const. }
$$

Also with a stiff fluid, we find the static self-similar solution as

$$
M=\frac{1}{2} a_{\omega}^{1 / 4}, \quad S=a_{\omega}^{1 / 4}, \quad \eta=\frac{1}{2} a_{\omega}^{-1 / 2}, \quad y=1,
$$

where the regularity condition (3.20) at the sonic point has been considered. Apparently, this solution seems unstable for early times but stable for late times. We can show, however, that this solution is always unstable. To demonstrate this explicitly, we change the coordinate system as

$$
w \equiv-2 \ln |u|+\ln R,
$$

for the scalar-field solution, and

$$
w \equiv \ln |t|, \quad R=a_{\omega}^{1 / 4} r,
$$

for the stiff-fluid solution. Then the line element for both cases is given in the static form as

$$
d s^{2}=-2 R^{2} d w^{2}+2 d R^{2}+R^{2}\left(d \theta+\sin \theta^{2} d \phi\right) .
$$

We should note that each of early-time and late-time solutions corresponds to the whole spacetime of the static solution through Eq. (5.3) or (5.4). Inversely, when Eq. (5.5) is given, it can be regarded as an early-time solution using the coordinate transformation Eq. (5.3) or (5.4). In this solution, the similarity horizon condition $\bar{g}=2 x$ or sonic point condition $V^{2}=1$ is satisfied everywhere. It implies that we can insert a kink mode everywhere. Then the evolution equation (2.30) or (3.37) for the kink mode perturbation holds along the radial null curve on which the discontinuity is inserted. Therefore, when we insert a kink mode perturbation, which vanishes inside and is discontinuous at some radius, it will blow up to infinity along the ingoing null curve $(w+\ln R=$ const) as time proceeds. In this sense, this solution is unstable. The situation is somewhat different for a perfect fluid with $P=k \rho$ for $0<k<1$, which will be described in Appendix [C]

\section{Horizons}

For self-similar stiff-fluid solutions, a sonic point may be a black hole event horizon, particle horizon or Cauchy horizon, simultaneously. Therefore, we can discuss the stability of these horizons. These horizons appear for latetime solutions. The black hole event horizon is characterized by an anti-transsonic point for late-time solutions. According to the result obtained here, $y_{\mathrm{s}}>1$ must be satisfied on the event horizon. Then the black hole event horizon corresponds to a saddle and is stable against the kink mode. The particle horizon and Cauchy horizon are characterized by transsonic points for late-time solutions. On these horizons we can have $0<y_{\mathrm{s}}<1 / 2$ or $1 / 2<y_{\mathrm{s}}<1$. These horizons correspond to nodes and are unstable if $0<y_{\mathrm{s}}<1 / 2$ while they are stable against the kink mode if $1 / 2<y_{\mathrm{s}}<1$. The above also applies to similarity horizons in equivalent scalar-field solutions using correspondence relation (4.11). 


\section{E. Other applications}

Nonanalytic but regular self-similar stiff-fluid solutions are possible only for $1 / 2<y_{\mathrm{s}}<1$. It is found that these solutions are unstable for early times but stable for late times. Anti-transsonic self-similar stiff-fluid solutions are possible only for $1<y_{\mathrm{s}}$. These solutions are unstable for early times but stable for late times. Again, the above also applies to equivalent scalar-field solutions using correspondence relation (4.11).

\section{SUMMARY}

We have investigated the stability of self-similar solutions with a scalar field and those with a stiff fluid in general relativity. The kink instability, which we have considered here, was studied in a Newtonian gas system [29] and in the Einstein-perfect-fluid system with the equation of state $P=k \rho$ (paper I). Since only the fluid system was considered in these previous works, it was not clear whether the kink instability is unique to fluid dynamics or not. The present work, for the first time, has shown the existence of kink instability in self-similar solutions of the Einstein-KleinGordon system. The most intriguing feature is that the kink instability grows exponentially in terms of $T=-\ln |u|$ for the scalar-field system and also in terms of $\tau=-\ln |t|$ for the stiff-fluid system, while it grows more rapidly and blows up to infinity at a finite moment before $t=0$ for the perfect-fluid system with $P=k \rho(0<k<1)$. In other words, the kink instability results in the reduction of the rank of differentiability and the formation of a shock wave before the singularity formation for a perfect fluid with $0<k<1$, while it does not for the scalar-field system and also for the stiff-fluid $(k=1)$ system until the singularity forms.

These systems have recently attracted attention in the context of critical phenomena in gravitational collapse. The present result shows that both the BCGN scalar-field solution and Evans-Coleman stiff-fluid solution are unstable against the kink mode. This implies that the latter solution, which was identified with a critical solution in the stiff-fluid collapse by Neilsen and Choptuik [23] and Brady et al. 26], cannot be a critical solution once we allow sufficiently small discontinuity in the density gradient field in the initial data sets; nor can the BCGN scalar-field solution be a critical solution once we allow sufficiently small discontinuity in the second-order derivative of the scalar field in the initial data sets. As another important application, we have shown the kink instability at the particle horizon of the flat Friedmann universe with a scalar field and with a stiff fluid, while the flat Friedmann collapse solution is stable against this mode.

\section{Acknowledgments}

TH is grateful to B J Carr and R Tavakol for helpful conversations. The authors greatly appreciate important comments by the anonymous referees, in particular on the estimate of the higher-order derivative term in Eqs. (2.29) and (3.35). TH was supported by JSPS Postdoctoral Fellowship for Research Abroad.

\section{APPENDIX A: ROBERTS SOLUTION}

In the Bondi coordinates (2.2), general solutions to Eqs. (2.13) - 2.17) with $\kappa=0$ are given by 34

$$
\begin{aligned}
& g=\frac{x}{\sqrt{\sigma^{2}+x^{2}}}, \\
& \bar{g}=\frac{\sqrt{\sigma^{2}+x^{2}}-2 \sigma^{2}}{x}, \\
& \phi=\frac{1}{4 \sqrt{\pi}} \ln \frac{\sqrt{\sigma^{2}+x^{2}}-\sigma}{\sqrt{\sigma^{2}+x^{2}}+\sigma},
\end{aligned}
$$

where $x$ is defined by Eq. (2.8), and the solutions are parametrized by $\sigma$ and referred to as Roberts solutions. This solution has been discussed in the context of cosmic censorship [34] and critical behaviour [39, 40].

For $\sigma=0$ this solution reduces to the Minkowski spacetime and we restrict $\sigma$ to $\sigma \neq 0$ below. Then, these solutions admit no timelike Killing vector. From Eq. (4.2), the energy density of the equivalent stiff fluid is formally given by

$$
\rho=\frac{1}{8 \pi R^{2}} \frac{\sigma^{2}\left(2 \sqrt{\sigma^{2}+x^{2}}-1\right)}{x^{2}} .
$$


We can see that the solution has a spacetime singularity at $x=0$. If and only if $0<|\sigma|<1 / 2$ this solution has a region around the centre given by

$$
0<x<x_{1}=\sqrt{\frac{1}{4}-\sigma^{2}},
$$

where the gradient of the scalar field is spacelike. At $x=x_{1}$ the solution has a null gradient of the scalar field. If and only if $0<|\sigma|<1 / 2$, the solution has a similarity horizon at

$$
x=x_{\mathrm{s}}=\sqrt{\frac{1}{4}-\sigma^{2}} .
$$

Therefore, for $0<|\sigma|<1 / 2$, the hypersurface $x=x_{1}$ where the gradient of the scalar field is null coincides with the similarity horizon $x=x_{\mathrm{s}}$. This implies that the stability analysis for self-similar stiff-fluid solutions developed in Sec. IIC cannot be used, although that for self-similar scalar-field solution developed in Sec. IC is still applicable.

See [41] for spherical and nonspherical perturbation with analyticity on the Roberts solution.

\section{APPENDIX B: HIGHER-ORDER DERIVATIVE TERM}

Let $f$ be continuous on $[0, \infty)$ and continuously differentiable on $(0, \infty)$ and have the limit value $\lim _{x \rightarrow+0} x f^{\prime}(x)$, where the prime denotes the differentiation with respect to the argument. When we define $g$ by $g(x) \equiv x f(x), g$ is continuous on $[0, \infty)$ with $g(0)=0$ and continuously differentiable on $(0, \infty)$ and have the limit value $\lim _{h \rightarrow+0} g(h) / h=$ $f(0)$. Then, from the mean value theorem, for any $a>0$, there exists $\xi(0<\xi<a)$, such that

$$
\frac{g(a)}{a}=g^{\prime}(\xi) .
$$

In terms of $f$, we have

$$
f(a)=\xi f^{\prime}(\xi)+f(\xi)
$$

When we take the limit $a \rightarrow+0, \xi$ also goes to zero. Since the left-hand side and the second term on the right-hand side approach the same limit value $f(0)$, the first term on the right-hand side goes to zero in this limit. This implies

$$
\lim _{x \rightarrow+0} x f^{\prime}(x)=0
$$

because we have assumed the existence of the above limit.

Applying the above proof, we can show that the right-hand side vanishes in Eq. (2.29) for the scalar-field system, where $f$ corresponds to $\delta j^{\prime}$, and in Eq. (3.35) for the perfect-fluid system, where $f$ corresponds to $\delta \eta^{\prime}$.

\section{APPENDIX C: STATIC SELF-SIMILAR PERFECT-FLUID SOLUTION}

We briefly review the static self-similar solution for a perfect fluid with the equation of state $P=k \rho$ for $0<k<1$. The case of $k=1$ is described in Sec. $\nabla \mathrm{C}$. We use $t$ and $r$ as the coordinates given by Eq. (3.3). For $0<k<1$, the line element in the static self-similar solution is written as

$$
d s^{2}=-\alpha_{k}^{2} R^{\frac{4 k}{1+k}} d w^{2}+\beta_{k}^{2} d R^{2}+R^{2}\left(d \theta^{2}+\sin ^{2} \theta d \phi\right),
$$

after the following coordinate transformation

$$
\begin{aligned}
& w= \begin{cases}-\frac{1+k}{1-k}(-t)^{\frac{1-k}{1+k}}+w_{0} & \text { for } \quad t<0 \\
\frac{1+k}{1-k} t^{\frac{1-k}{1+k}}+w_{0} & \text { for } t>0\end{cases} \\
& R=\gamma_{k} r,
\end{aligned}
$$

where $\alpha_{k}, \beta_{k}$ and $\gamma_{k}$ are some positive constants which can depend on $a_{\sigma}, a_{\omega}$ and $k$, and $w_{0}$ is an arbitrary constant. In the above, $w$ increases from $-\infty$ to $+\infty$ as $t$ increases from $-\infty$ to $+\infty$. Therefore, the static self-similar solution 
consists of both the early-time solution and the late-time solution. We can identify an arbitrary value of $w$ with $t=0$ because of the arbitrary constant $w_{0}$. Therefore any value of the time coordinate $w$ can be regarded as in early time and also in late time. This is essential to the instability of the static self-similar solution as is discussed in paper I.

[1] Cahill M E and Taub A H 1971 Comm. Math. Phys. 211

[2] Goliath M, Nilsson U and Uggla C 1998 Class. Quantum Grav. 15 167;

Goliath M, Nilsson U and Uggla C 1998 Class. Quantum Grav. 152841

[3] Carr B J and Coley A A 2000 Phys. Rev. D 62 044023;

Carr B J and Coley A A 2000 Class. Quantum Grav. 174339

[4] Carr B J, Coley A A, Goliath M, Nilsson U and Uggla C 2000 Phys. Rev. D 61081502 ; Carr B J, Coley A A, Goliath M, Nilsson U and Uggla C 2001 Class. Quantum Grav. 18303

[5] Wainwright J and Ellis G F R "Dynamical Systems in Cosmology" (Cambridge University Press, Cambridge, England, 1997)

[6] Coley A A 1999 gr-qc/9910074

[7] Carr B J and Hawking S W 1974 Mon. Not. R. Astr. Soc. 168399

[8] Lin D N C, Carr B J and Fall S M 1976 Mon. Not. R. Astr. Soc. 17751

[9] Bicknell G V and Henriksen R N 1978 ApJ 219 1043;

Bicknell G V and Henriksen R N 1978 ApJ 225237

[10] Ori A and Piran T 1987 Phys. Rev. Lett. 59 2137;

Ori A and Piran T 1988 Gen. Rel. Grav. 207

[11] Ori A and Piran T 1990 Phys. Rev. D 421068

[12] Foglizzo T and Henriksen R N 1993 Phys. Rev. D 484645

[13] Carr B J and Coley A A 1999 Class. Quantum Grav. 16 R31-R71

[14] Carr B J 1993 unpublished

[15] Harada T and Maeda H, 2001 Phys. Rev. D 63084022

[16] Harada T 1998 Phys. Rev. D 58104015

[17] Choptuik M W 1993 Phys. Rev. Lett. 709

[18] Hamadé S H and Stewart J M 1996 Class. Quantum. Grav. 13497

[19] Evans C R and Coleman J S 1994 Phys. Rev. Lett. 721782

[20] Koike T, Hara T and Adachi S 1995 Phys. Rev. Lett. 745170

[21] Gundlach C 1995 Phys. Rev. Lett. 75 3214;

Gundlach C 1997 Phys. Rev. D 55695

[22] Maison D 1996 Phys. Lett. B 366 82;

Koike T, Hara T and Adachi S 1999 Phys. Rev. D 59104008

[23] Neilsen D W and Choptuik M W 2000 Class. Quantum Grav. 17 733; Neilsen D W and Choptuik M W 2000 Class. Quantum Grav. 17761

[24] Maeda H and Harada T 2001 Phys. Rev. D 64 124024;

Harada T, Maeda H and Semelin B 2003 Phys. Rev. D 67084003

[25] Madsen M S 1988 Class. Quantum Grav. 5 627;

Carr B J and Goymer C A 1999 Prog. Theor. Phys. Suppl. 136321

[26] Brady P R, Choptuik M W, Gundlach C and Neilsen D W 2002 Class. Quantum Grav. 196359

[27] Gundlach C 2003 Phys. Rep. 376339

[28] Hawking S W and Ellis G F R “The large scale structure of space-time” (Cambridge University Press, Cambridge, England, 1973)

[29] Ori A and Piran T 1988 MNRAS 234821

[30] Harada T 2001 Class. Quantum Grav. 184549

[31] Wald R M "General Relativity" (University of Chicago Press, Chicago, United States of America, 1983)

[32] Christodoulou D 1994 Annals of Math 140607

[33] Brady P 1995 Phys. Rev. D 514168

[34] Roberts M D 1989 Gen. Rel. Grav. 21907

[35] Misner C W and Sharp D H 1964 Phys. Rev. D 136571

[36] Equation (3.32) of paper I contains a trivial typo.

[37] The existence of the finite limit value for $\delta \dot{\eta}^{\prime}$ is not explicitly assumed in paper I.

[38] Equation (3.35) corresponds to Eq. (4.15) in paper I, where the right-hand side is ignored without caution.

[39] Brady P 1994 Class. Quantum Grav. 111255

[40] Oshiro Y, Nakamura K and Tomimatsu A 1994 Prog. Theor. Phys. 911265

[41] Frolov A V 1997 Phys. Rev. D 56 6433;

Frolov A V 1999 Phys. Rev. D 59 104011;

Frolov A V 2000 Phys. Rev. D 61084006 